\title{
Twist and writhe of $\delta$ active region magnetic fields
}

\author{
Lirong Tian ${ }^{1} \&$ Jing Yang ${ }^{2}$ \\ ${ }^{1}$ National Astronomical Observatories, Chinese Academy of Sciences, \\ Beijing 100012, China (tlr@ns.bao.ac.cn); ${ }^{2}$ Astronomical Department, \\ Beijing Normal University, Beijing 100875, China
}

Twist is a parameter to measure turning of the field lines around axis of the flux tube. Observations show that each active region (AR) has a pronounced overall twist, following hemispheric helicity rule (Pevtsov et al. 1995; Bao \& Zhang 1998). $\alpha_{\text {best }}$, best-fit single value for a whole AR, is used to characterize the overall twist. Writhe is a measure of the spatial turning of the axis of the flux tube. It is described by systematic tilt angle of an AR, an angle of joined line of opposite main polarities with respect to the equator. Observations show that most ARs follow Joy's Law. The relationship between the twist and writhe is important to studying origin of the twist and solar activities. Canfield \& Pevtsov (1998) found the same handedness of the twist as that of writhe, using 99 ARs observed at MSO. However, Tian et al. (2001) found opposite handedness of them for 286 ARs taken at HSOS, among which only $19 \%$ have $\delta$ magnetic configuration. López Fuentes et al. (2003) also obtained opposite handedness for 22 ARs measured again at MSO, among which $41 \%$ have $\delta$ magnetic class. Most recently, Tian \& Liu (2003) found that the twist and the writhe have same handedness for major flare-producing ARs.

A kink hypothesis predict that sign of the twist and writhe should be same where a kink instability has developed and for $\delta$ ARs (Linton et al. 1999; Fisher et al. 2000). Therefore, the motivation of this work is to investigate if kink instability prefers to occur in $\delta$ ARs. We select 104 ARs with $\delta$ magnetic configuration from 1996 to 2002 . SOHO/MDI $96 \mathrm{~m}$ full disk magnetograms are used to calculate the tilt angle and vector magnetograms from HSOS are used to calculate the $\alpha_{\text {best }}$. In our sample, most ARs have two main polarities and a dominant sign of the $\alpha_{b e s t}$ unchanged in several days. Three sets of magnetograms near the central meridian are used to obtain a mean value and error for the tilt and $\alpha_{\text {best }}$. The signs of the mean values denote the handedness of the writhe and twist: the positive/negative values corresponding to the right/left writhe( twist). The results show that (1) only $54 \%$ of the ARs follow Joy's law and $50 \%$ obey the hemispheric helicity rule; (2) $67 \%$ (35.9\% with positive tilts and $\alpha_{\text {best }}$ and $31.1 \%$ with negative tilts and $\alpha_{\text {best }}$ ) of the ARs have the same handedness of the twist and writhe. A least-square fit for all 104 ARs demonstrates a positive correlation (correlation coefficient, 0.322) between them with more than $99 \%$ confidence level. Thus, we further believe that the different relationship between the twist and writhe, obtained in above papers, is due to difference of sample selection: opposite handedness for ARs with simple magnetic configuration and producing less flares and same handedness for ARs with complex magnetic configuration and producing more major flares. 


\section{References}

Pevtsov et al. 1995, ApJ, 440, 109

Bao \& Zhang, 1998, ApJ, 496, 43

Canfield \& Pevtsov 1998, ASP Conf. Ser. 140, 131

Tian et al. 2002, Sol. Phys. 209, 361

Lopez-Fuentes et al. 2003, A\&A, 397, 305

Tan \& Liu 2002, A\&A, 407, 13

Linton et al. 1999, ApJ, 522, 1190

Fisher et al. 2000, Sol. Phys. 192, 119 